\title{
Involvement of ER- $\alpha 36$, Src, EGFR and STAT5 in the biphasic estrogen signaling of ER-negative breast cancer cells
}

\author{
XIN-TIAN ZHANG ${ }^{1}$, LING DING ${ }^{2}$, LIAN-GUO KANG ${ }^{1}$ and ZHAO-YI WANG ${ }^{1}$ \\ ${ }^{1}$ Department of Medical Microbiology and Immunology, Creighton University Medical School, \\ Omaha, NE, USA; ${ }^{2}$ Department of Oncology, Second Affiliated Hospital, Zhejiang \\ University School of Medicine, Hangzhou, Zhejiang, P.R. China
}

Received December 20, 2011; Accepted February 10, 2012

DOI: $10.3892 /$ or.2012.1722

\begin{abstract}
It is well established that estrogen is a potent mitogen in cells expressing estrogen receptors (ER). However, a large body of evidence has demonstrated that the effects of mitogenic estrogen signaling exhibit a non-monotonic or biphasic, dose-response curve; estrogen at low concentrations, elicits a mitogenic signaling pathway to stimulate cell proliferation, while at high concentrations, estrogen inhibits cell growth. The molecular mechanism underlying this paradoxical effect of estrogen on cell proliferation remains largely unknown. Recently, we reported that ER- $\alpha 36$, a variant of ER- $\alpha$, mediates mitogenic estrogen signaling in ER-negative breast cancer cells. Here, we investigated the molecular mechanisms underlying the biphasic estrogen signaling in MDA-MB-231 and MDA-MB-436 ER-negative breast cancer cells. We found that $17 \beta$-estradiol (E2 $\beta$ ) at $1 \mathrm{nM}$ induced the phosphorylation of Src-Y416, an event that activates Src, while at $5 \mu \mathrm{M}$ failed to induce Src-Y416 phosphorylation but induced Src-Y527 phosphorylation an event that inactivates Src. E2 $\beta$ at $1 \mathrm{nM}$, but not at $5 \mu \mathrm{M}$, also induced phosphorylation of MAPK/ERK and activated Cyclin D1 promoter activity through the Src/EGFR/STAT5 pathway. Knockdown of ER- $\alpha 36$ abrogated the biphasic estrogen signaling in these cells. Our results thus indicate that in ER-negative breast cancer cells Src functions as a switch in ER- $\alpha 36$-mediated biphasic estrogen signaling through the EGFR/STAT5 pathway.
\end{abstract}

\section{Introduction}

It is well known that the diverse physiological functions of estrogens are mediated by specific receptors, ER- $\alpha$ and ER- $\beta$, which

Correspondence to: Dr Zhao-Yi Wang, Department of Medical Microbiology and Immunology, Creighton University Medical School, Criss III, Room 355, 2500 California Plaza, Omaha, NE 68178, USA E-mail: zywang@creighton.edu

Key words: estrogen, biphasic response, ER- $\alpha 36$, Src, EGFR, STAT5, ER-negative breast cancer cells share a common structural architecture (1); the N-terminal A/B domains that encodes a ligand-independent activation function (AF-1), the $\mathrm{C}$ domain functions as the DNA-binding domain and the $\mathrm{D} / \mathrm{E} / \mathrm{F}$ or ligand-binding domain that encodes a liganddependent transactivation function (AF-2).

ERs are well-known ligand-activated transcription factors that stimulate target gene transcription, which mediates diverse activities of estrogen signaling (1). However, despite the clarity with which ERs have been shown to act as transcription factors, it became apparent that not all of the physiological effects mediated by estrogens are accomplished through a direct effect on gene transcription. Another estrogen signaling pathway (also known as a non-classic, non-genomic or membrane-initiated estrogen signaling pathway) exists that involves cytoplasmic proteins, growth factors and other membrane-initiated signaling pathways $(2,3)$.

Previously, we cloned ER- $\alpha 36$, a $36-\mathrm{kDa}$ variant of ER- $\alpha$. ER- $\alpha 36$ is mainly expressed on the cell surface and mediates membrane-initiated or non-genomic estrogen signaling $(4,5)$. ER- $\alpha 36$ lacks both the AF-1 and AF-2 domains of the 66-kDa ER- $\alpha($ ER- $\alpha 66)$ and possesses an altered ligand-binding domain. ER- $\alpha 36$ lacks intrinsic transcriptional activity but inhibits transcriptional transactivation activities of both ER- $\alpha 66$ and ER- $\beta$ (5). The transcription of ER- $\alpha 36$ is initiated from and regulated by a promoter located in the first intron of the ER- $\alpha 66$ gene (6). Thus, ER- $\alpha 36$ expression is regulated differently from ER- $\alpha 66$, consistent with the previous findings that ER- $\alpha 36$ is expressed in specimens from ER-negative patients and established ER-negative breast cancer cells (7-9). We recently found that ER- $\alpha 36$ mediates mitogenic estrogen signaling in ER-negative breast cancer MDA-MB-231 and MDA-MB-436 cells that express endogenous ER- $\alpha 36$, but lack ER- $\alpha 66$ expression (10).

A large body of evidence demonstrated that the effects of mitogenic estrogen signaling exhibit a non-monotonic or biphasic dose-response curve (inverted U-shaped) (11-14); estrogen at low concentrations, elicits mitogenic signaling to stimulate cell proliferation, while at high concentrations, estrogen inhibits cell growth and even induces cell apoptosis. However, the molecular basis for this paradoxical effect of estrogen on the cell proliferation remains largely unknown. A 
variety of possible mechanisms have been proposed to explain the inhibitory effects of high-dose estrogens such as effects on cell membrane $(15,16)$, cell cycle arrest $(17)$ and the interaction of estrogens with the mitotic spindle $(18,19)$. In addition, the involvement of the non-genomic estrogen signaling in the biphasic estrogen response has also been proposed (20); the activation of a cascade of protein kinases may modulate the genomic activities of estrogens in opposite directions through phosphorylation of ERs or co-regulators, resulting in biphasic responses of estrogen signaling.

High-dose estrogen therapy using pharmacological concentrations of estrogen (at $\mu \mathrm{M}$ range) were frequently used for treatment of advanced breast cancer in pre- and post-menopausal women from the 1950s until the introduction of the safer antiestrogen tamoxifen in the 1970s. Since the introduction of tamoxifen, which almost completely eliminated high-dose estrogen therapy, there was no enthusiasm to study the underlying mechanisms of high-dose estrogen therapy. However, recent laboratory findings indicated that growth of tamoxifen-resistant breast cancer cells is inhibited by $17 \beta$-estradiol (E2 $\beta$ ) and physiological concentrations of E2 $\beta$ induce apoptosis in long-term estrogen deprived (LTED) breast cancer cells in vivo and in vitro (21-26). Thus, better understanding of underlying mechanisms of the paradoxical effects of estrogen will enhance the effects of estrogen therapy in the treatment of antiestrogen-resistant breast cancer.

Recently, we reported that ER- $\alpha 36$ mediates mitogenic estrogen signaling through the EGFR/Src signaling in ER-negative breast cancer MDA-MB-231 and MDA-MB-436 cells. In the current study, we investigated the molecular mechanisms underlying biphasic estrogen signaling in these ER-negative breast cancer cells and reveal the involvement of the Src/EGFR/STAT5 signaling pathway in the biphasic estrogen signaling.

\section{Materials and methods}

Chemicals and antibodies. $17 \beta$-estradiol (E2 $\beta)$ was purchased from Sigma Chemicals Co. (St. Louis, MO). The Src inhibitor dasatinib was obtained from LC Laboratories (Woburn, MA). The Src inhibitor PP2 and the PI3K inhibitor LY294002 were from Tocris Bioscience (Ellisville, MO). Anti-phosphoEGFR and -Src antibodies, anti-EGFR and -Src antibodies, anti-phospho-p44/42 ERK (Thr202/Tyr204) (197G2) mouse monoclonal antibody (mAb) and anti-p44/42 ERK (137F5) rabbit $\mathrm{mAb}$ were purchased from Cell Signaling Technology (Boston, MA). An antibody against Cyclin D1 was purchased from Santa Cruz Biotechnology (Santa Cruz, CA).

Cell culture, treatment and growth assay. MDA-MB-231 and MDA-MB-436 cells were obtained from the American Type Culture Collection (ATCC, Manassas, VA). All parental and derivative cells were maintained at $37^{\circ} \mathrm{C}$ in a $10 \% \mathrm{CO}_{2}$ atmosphere in DMEM and $10 \%$ fetal calf serum in a humidified incubator. For E2 $\beta$ treatment, cells were maintained in phenol red-free media with $2.5 \%$ dextran-charcoal-stripped fetal calf serum (HyClone, Logan, UT) for 2-3 days and then in serumfree medium for $24 \mathrm{~h}$ before experimentation. For ERK activation assays, cells were treated with vehicle (ethanol) and indicated concentrations of E2 $\beta$. To test the effects of different inhibitors, all inhibitors were added $10 \mathrm{~min}$ before E2 $\beta$ addition.

To examine cell growth in the presence or absence of different concentrations of E2 $\beta$, cells maintained for three days in phenol red-free DMEM plus 2.5\% dextran-charcoal-stripped fetal calf serum were treated with different concentrations of E2 $\beta$ or ethanol vehicle as a control. The cells were seeded at $1 \times 10^{4}$ cells per dish in $60-\mathrm{mm}$ dishes and the cell numbers were determined using the ADAM automatic cell counter (Digital Bio., Korea) after 12 days. Five dishes were used for each treatment and experiments were repeated $>3$ times.

Cell lines with ER- $\alpha 36$ expression knocked down by the shRNA method in MDA-MB-231 and MDA-MB-436 cells were generated as previously described (27).

Plasmids, DNA transfection and luciferase assay. The expression vectors for a dominant-negative mutant of Src (pCMV5/ SrcK295) and a constitutively active mutant of Src (pCMV5/ SrcY527F) were obtained from Dr Yun Qiu (Department of Pharmacology and Experimental Therapeutics, University of Maryland School of Medicine). Dr Linda Schuler (Department of Comparative Biosciences, University of Wisconsin-Madison) kindly provided the luciferase reporter plasmids of the Cyclin D1 promoter (pl-963) carrying GAS1 and 2 mutations. Two naturally occurring dominant-negative STAT5 mutants, Stat5a $\Delta 713$ and Stat5a $\Delta 740$ were provided by Dr Hiroko Yamashita (Department of Surgery II, Nagoya City University). The wild-type luciferase reporter plasmid of the Cyclin D1 promoter, Cyclin D1 pl-963 was obtained from Dr Chris Albanese (Departments of Oncology and Pathology, Georgetown University Medical Center). The 4XM67 pTATATK-luciferase reporter plasmid was purchased from Addgene (Cambridge, MA). Cells were all co-transfected with a cytomegalovirus-driven Renilla luciferase plasmid, pRL-CMV (Promega, Madison, WI) to establish transfection efficiency. Twenty-four hours after transfection, cells were treated with vehicle or E2 $\beta$, together with or without the indicated inhibitors for $24 \mathrm{~h}$. Forty-eight hours after transfection, cell extracts were prepared and luciferase activities were determined and normalized using the Dual-Luciferase Assay System (Promega) and a TD 20/20 Luminometer (Turner BioSystems, Inc., Sunnyvale, CA) as instructed by the manufacturer.

Western blot analysis. For immunoblot analysis, cells washed with PBS were lysed with lysis buffer $(50 \mathrm{mM}$ Tris- $\mathrm{HCl}$, $\mathrm{pH}$ 8.0, $150 \mathrm{mM} \mathrm{NaCl}, 0.25 \mathrm{mM}$ EDTA pH 8.0, 0.1\% SDS, $1 \%$ Triton $\mathrm{X}-100,50 \mathrm{mM} \mathrm{NaF}$ ) supplemented with protease and phosphatase inhibitors (Sigma). The protein amounts were measured using the DC protein assay kit (Bio-Rad Laboratories, Hercules, CA). The same amounts of the cell lysates were boiled for $5 \mathrm{~min}$ in loading buffer and separated on an SDS-PAGE gel. After electrophoresis, the proteins were transferred to a PVDF membrane (Bio-Rad Laboratories). The membranes were probed with various primary antibodies, HRP-conjugated secondary antibodies and visualized with enhanced chemiluminescence (ECL) detection reagents (GE Healthcare, Piscataway, NJ).

Statistical analysis. Data are expressed as the mean \pm standard error (SE) using the GraphPad InStat software program 
A

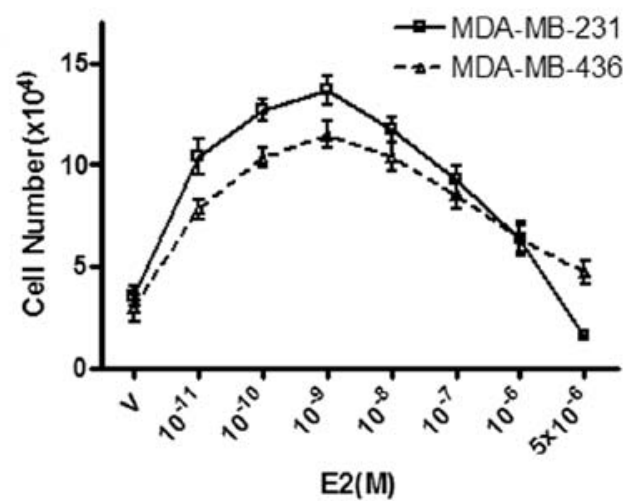

B

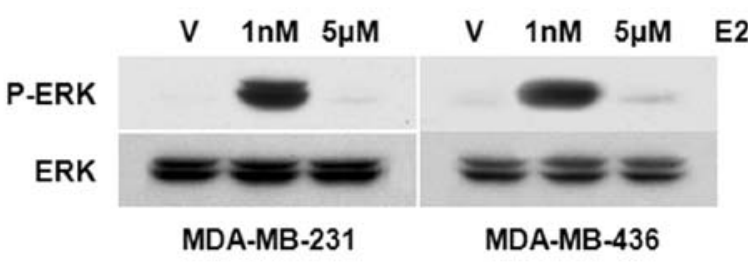

C

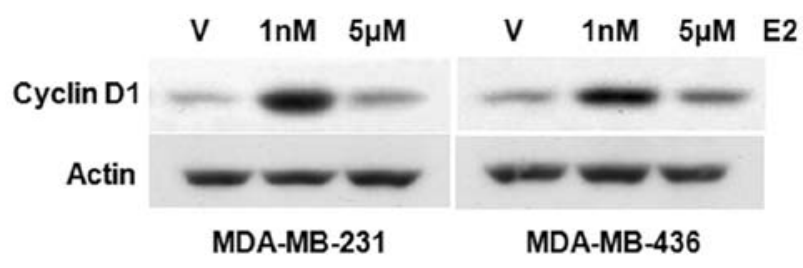

Figure 1. ER-negative breast cancer cells exhibit biphasic estrogen signaling. (A) The effects of E2 $\beta$ on the proliferation rate of MDA-MB-231 and MDA-MB-436 cells. Cells maintained for three days in phenol red-free DMEM plus $2.5 \%$ dextran-charcoal-stripped fetal calf serum were treated with indicated concentrations of E2 $\beta$ or ethanol vehicle $(V)$ as a control. The cell numbers were determined using an automatic cell counter after 12 days. Five dishes were used for each concentration and experiments were repeated more than 3 times. The mean cell number \pm SE are shown. (B) Dosedependent pattern of E2 $\beta$-stimulated phosphorylation of the MAPK/ERK1/2 in MDA-MB-231 and MDA-MB-436 cells. Starved cells were treated with indicated doses of E2 $\beta$ for $10 \mathrm{~min}$. Western blot analysis was performed to assess induction of ERK1/2 phosphorylation. The experiment was repeated more than 3 times. The representative results are shown. (C). Dose-dependen induction of Cyclin D1 by E2 $\beta$ in MDA-MB-231 and MDA-MB- 436 cells. The experiment was repeated more than 3 times. Representative results are shown.

(GraphPad Software, La Jolla, CA, USA). The Tukey-Kramer Multiple Comparisons Test was used, and the significance was set at $\mathrm{P}<0.05$.

\section{Results}

ER-negative breast cancer cells exhibit biphasic estrogen signaling. Recently, we reported that ER- $\alpha 36$ mediates mitogenic estrogen signaling in ER-negative breast cancer cells, MDA-MB-231 and MDA-MB-436, both of which lack expression of ER- $\alpha 66$ but express high levels of endogenous ER- $\alpha 36$ (10). To probe the underlying mechanisms of ER- $\alpha 36$-mediated-estrogen signaling, we first determined the growth rate of these two cell lines in response to different concentrations of E2 $\beta$. As shown in Fig. 1A, the ER-negative breast cancer cells treated with low concentrations $(<100 \mathrm{nM})$

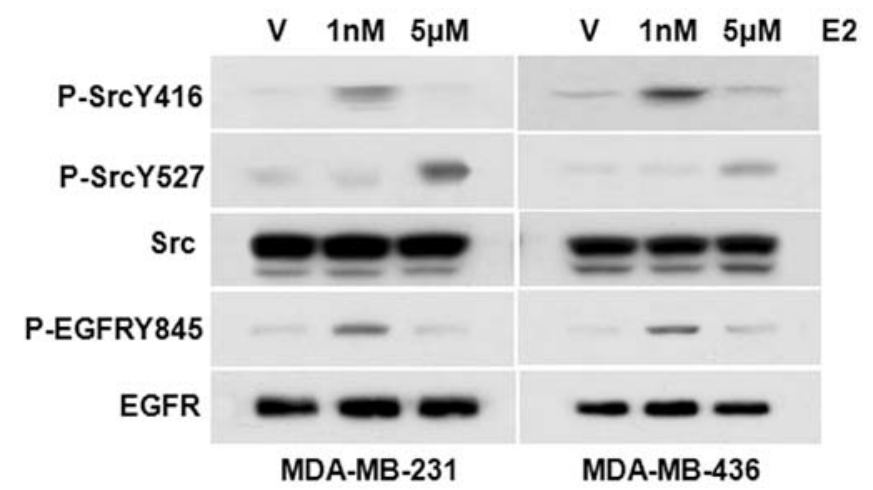

Figure 2. Different concentrations of E2 $\beta$ induce Src phosphorylation at distinct residues. Western blot analysis of the effects of different concentrations of E2 $\beta$ on the phosphorylation levels of EGFR-Y845, Src-Y416 and Src-Y527 in MDA-MB-231 and MDA-MB-436 cells

E2 $\beta$ exhibited an increased growth rate compared with cells treated with vehicle. The dose-response curves of E2 $\beta$ exhibited a biphasic pattern; increasing concentrations initially stimulated cell growth but failed to do so at higher concentrations (Fig. 1A). Our data thus indicate that E2 $\beta$ exhibited a non-monotonic or biphasic dose-response curve in these ER-negative breast cancer cells.

E2 $\beta$ induces biphasic activation of the MAPK/ERK and Cyclin D1 expression in ER-negative breast cancer cells. We then examined estrogen-induced phosphorylation of the MAPK/ERK1/2, a typical non-genomic estrogen-signaling event, in these two cell lines. We treated cells with E2 $\beta$ at different concentrations ( $1 \mathrm{nM}$ and $5 \mu \mathrm{M}$ ) for $30 \mathrm{~min}$. Western blot analysis with a phospho-specific ERK1/2 antibody was performed to assess the phosphorylation levels of ERK1/2. As shown in Fig. 1B, we found that E2 $\beta$ was able to induce the activation of MAPK/ERK at $1 \mathrm{nM}$ but not at $5 \mu \mathrm{M}$ in both ER-negative breast cancer cell lines, consistent with the biphasic growth pattern of the dose-response curves of these cells to E2 $\beta$. To determine whether $5 \mu \mathrm{M}$ estrogen failed to activate the MAPK/ERK or inhibited the ERK activation, we examined the effects of $5 \mu \mathrm{M}$ E2 $\beta$ on the ERK activation induced by EGF. We found that estrogen at $5 \mu \mathrm{M}$ failed to inhibit the ERK activation induced by EGF (data not shown), indicating that E2 $\beta$ at high concentrations failed to induce ERK activation. Additionally, we found a biphasic induction pattern of Cyclin D1 expression in the cells treated with two different concentrations of E2 $\beta$ (Fig. 1C).

Src/EGFR/STAT5 are involved in biphasic estrogen signaling. Recently, we reported that in these ER-negative breast cancer cells, estrogen induced phosphorylation of Src-Y416 and also phosphorylation of EGFR-Y845, a site phosphorylated by Src (10). We then examined the phosphorylation status of Src-Y416 and EGFR-Y845 in these cells treated with different concentrations of E2 $\beta$. Fig. 2 shows that in MDA-MB-231 and MDA-MB-436 cells, $1 \mathrm{nM}$ E2 $\beta$ elicited phosphorylation of Src-Y416 and EGFR-Y845 while failed to do so at $5 \mu \mathrm{M}$. Intriguingly, $5 \mu \mathrm{M}$ of $\mathrm{E} 2 \beta$ strongly induced phosphorylation of Src-Y527, an event associated with inactivation of Src 
A
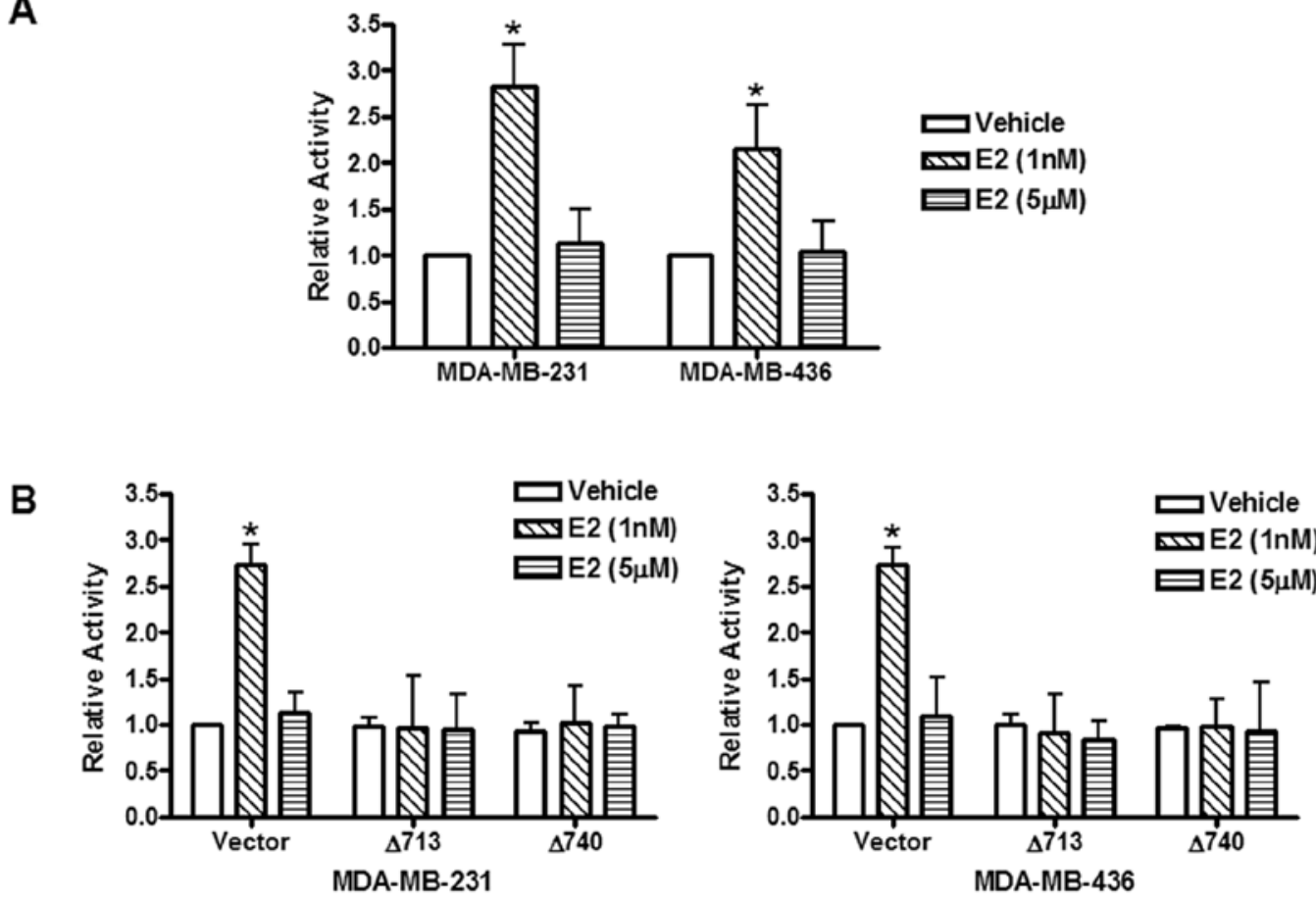

Figure 3. E2 $\beta$ induces biphasic STAT5 activities in ER-negative breast cancer cells. (A) ER-negative breast cancer cells were transfected with the luciferase reported plasmid 4XM67 TATA-TK-Luc that containing four copies of STAT-binding sites upstream of the minimal TK promoter. Transfected cells were treated with vehicle (ethanol), $1 \mathrm{nM}$ or $5 \mu \mathrm{M}$ of E2 $\beta$. The luciferase activities were assayed and normalized using a cytomegalovirus-driven Renilla luciferase plasmid. Columns, means of the relative luciferase activity from 4 independent experiments. Luciferase activity in transfected cells treated with vehicle is arbitrarily set as 1.0; bars, $\mathrm{SE}$. "P $<0.05$, for cells treated with vehicle vs. $1 \mathrm{nM}$ of E2 $\beta$. (B) Cells were transfected with the 4XM67 TATA-TK-Luc reporter together with an empty expression vector (vector) and the expression vectors of two dominant-negative STAT5a mutants carrying truncations at their C-terminal (STAT5a $\Delta 713$ and STAT5a $\Delta 740$ ) before treatment with vehicle (ethanol), $1 \mathrm{nM}$ or $5 \mu \mathrm{M}$ of E2 $\beta$. Columns, means of the relative luciferase activity from 3 independent experiments. Luciferase activity of cells co-transfected with an empty expression vector and treated with vehicle is arbitrarily set as 1.0; bars, SE. ${ }^{*} \mathrm{P}<0.05$, for cells treated with vehicle vs. $1 \mathrm{nM}$ of E2 $\beta$.

activity, which was not observed in the cells treated with $1 \mathrm{nM}$ of E2 $\beta$ (Fig. 2). These results suggest that E2 $\beta$ at low concentrations induces phosphorylation of Src-Y416 and activating Src whereas at high concentrations, it induces Src-Y527 phosphorylation inactivating Src activity.

It was reported that the signal transducer and activator of transcription 5b (STAT5b), c-Src and EGFR play integral roles in estrogen-stimulated proliferation of ER-positive breast cancer cells (27); estrogen-induced Src activation and Src-dependent phosphorylation of EGFR-Y845 recruit STAT5 as a downstream effector of phosphorylated EGFR-Y845. To examine whether STAT5 is involved in the observed biphasic estrogen signaling, we transfected MDA-MB-231 and MDA-MB-436 cells with the 4XM67 pTATA-TK-luciferase reporter plasmid that contains four copies of the STATbinding site and treated the cells with $\mathrm{E} 2 \beta$ at $1 \mathrm{nM}$ and $5 \mu \mathrm{M}$. We found that $1 \mathrm{nM} \mathrm{E} 2 \beta$ potently activated the promoter activity of the reporter plasmid while $5 \mu \mathrm{M} \mathrm{E} 2 \beta$ failed to do so (Fig. 3), suggesting that E2 $\beta$ at low concentrations was able to activate STAT protein-mediated transcription. To confirm the involvement of STAT5, we also included two naturally occurring dominant-negative mutants of STAT5 described before (28) in the transient transfection assays, and found that both dominant-negative mutants of STAT5 potently inhibited $1 \mathrm{nM}$ E2 $\beta$ induced promoter activity, indicating that STAT5 is involved in the biphasic estrogen signaling (Fig. 3).
Src is involved in biphasic Cyclin D1 expression in response to different concentrations of estrogen. In the experiments described above, we observed that the cells treated with different concentrations of estrogen also exhibited biphasic patterns of Cyclin D1 induction. Previously, it was reported that prolactin activated Cyclin D1 promoter activity through activation of STAT5b proteins and their interaction with the STAT binding-sites located in the Cyclin D1 promoter (29). We decided to determine whether the Src signaling pathway is involved in Cyclin D1 induction by E2 $\beta$ in these cells. We first assessed whether the Src inhibitors PP2 and dasatinib were able to inhibit Cyclin D1 induction by $1 \mathrm{nM}$ of E2 $\beta$. Cells were treated with $1 \mathrm{nM} \mathrm{E} 2 \beta$ and together with PP2, dasatinib, the EGFR inhibitor AG1478 or the PI3K inhibitor LY294002 and western blot analysis was performed to examine Cyclin D1 expression. E2 $\beta$-induced Cyclin D1 expression was strongly blocked by both Src inhibitors but weakly by AG1478 and LY294002 (Fig. 4A), suggesting that Src is involved in E2 $\beta$ induction of Cyclin D1 expression. To further confirm whether the Src/EGFR/STAT5 pathway is involved in estrogen induction of the Cyclin D1 promoter activity, we first transfected both cell lines with a human Cyclin D1 promoter-luciferase construct and then treated transfected cells with $1 \mathrm{nM}$ or $5 \mu \mathrm{M}$ E2 $\beta$. We found that $1 \mathrm{nM}$ E2 $\beta$ was able to induce Cyclin D1 promoter activity about 2-fold whereas $5 \mu \mathrm{M}$ E2 $\beta$ failed to induce Cyclin D1 


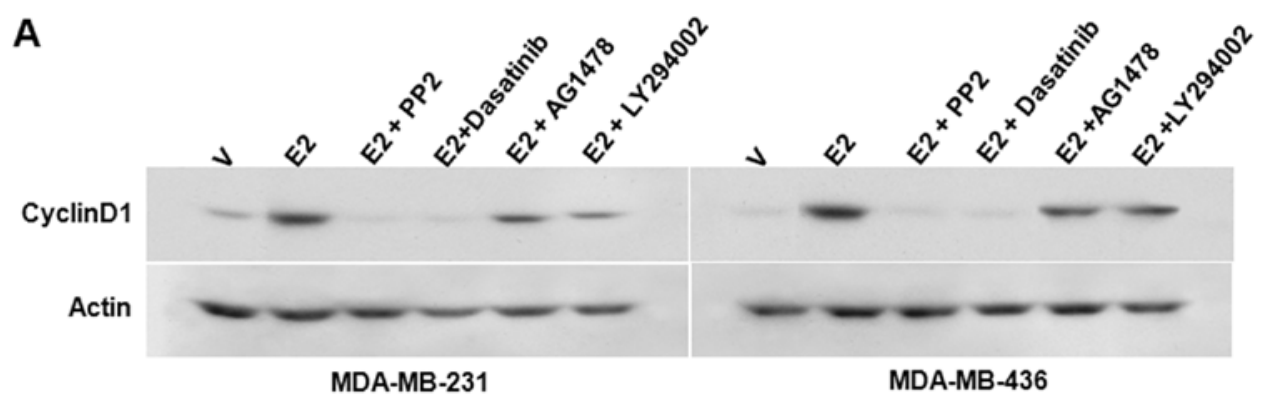

B
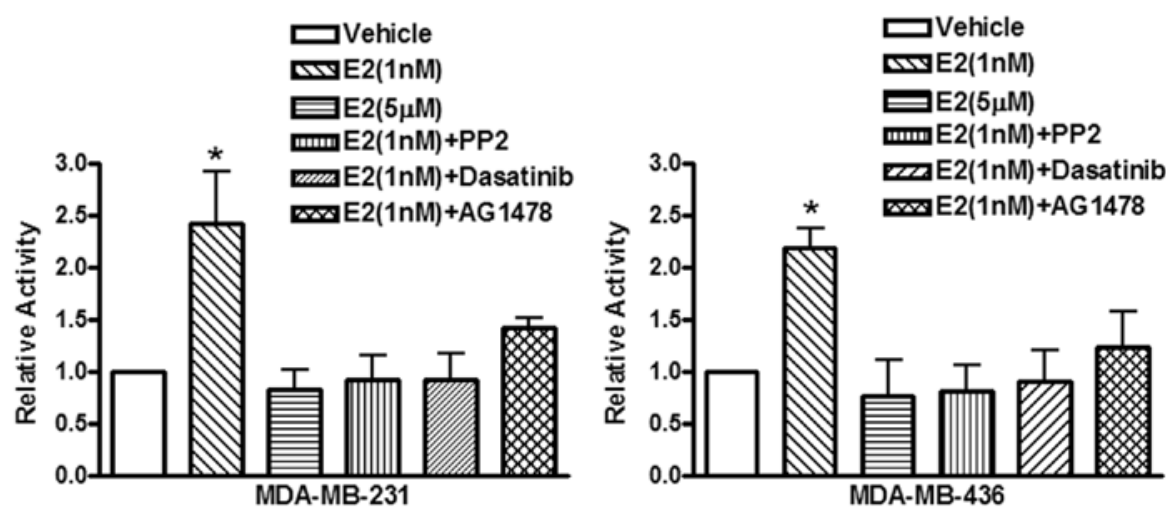

C
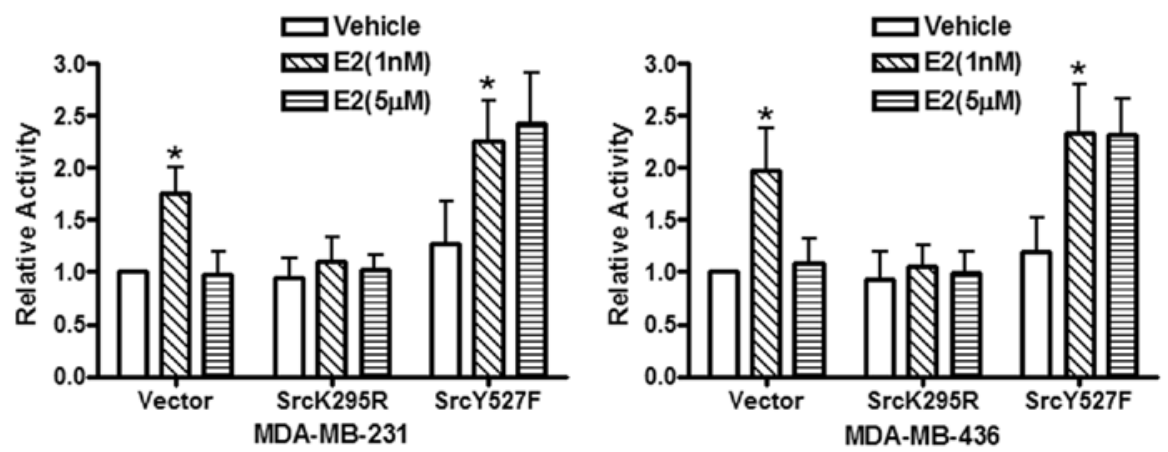

Figure 4. Src is involved in estrogen induction of Cyclin D1. (A) Western blot analysis of Cyclin D1 expression in MDA-MB-231 and MDA-MB-436 cells. Cells were treated with ethanol vehicle (V) and E2 $\beta$ alone or together with the Src inhibitors PP2 and dasatinib, the EGFR inhibitor AG1478 and the PI3K inhibitor LY294002. Cell lysates were analyzed with anti-Cyclin D1 antibody; anti-actin antibody was used to ensure equal loading. The experiment was repeated 3 times and the representative results are shown. (B) Src inhibitors inhibit E2 $\beta$ induction of Cyclin D1 promoter activity. ER-negative breast cancer cells were transfected with the luciferase reported plasmid Cyclin D1 pl-963 that containing a luciferase gene driven by the Cyclin D1 promoter. Transfected cells were treated with vehicle (ethanol), $1 \mathrm{nM}$ or $5 \mu \mathrm{M}$ of E2 $\beta$ and $1 \mathrm{nM} \mathrm{E} 2 \beta$ together with different inhibitors. The luciferase activities were assayed and normalized using a cytomegalovirus-driven Renilla luciferase plasmid. Columns, means of the relative luciferase activity in cells treated with vehicle that is arbitrarily set as 1.0 from 4 independent experiments; bars, SE. "P $<0.05$, for cells treated with vehicle vs. $1 \mathrm{nM}$ of E2 $\beta$. (C) The involvement of Src in E2 $\beta$ induction of Cyclin D1 promoter activity. Cells were transfected with the luciferase reported plasmid Cyclin D1 pl-963 together with an empty expression vector or Src mutants, a dominant-negative mutant (SrcK295R) and a constitutively active mutant (SrcY527F). Transfected cells were treated with vehicle (ethanol), $1 \mathrm{nM}$ or $5 \mu \mathrm{M}$ of E2 $\beta$. The luciferase activities were assayed and normalized using a cytomegalovirus-driven Renilla luciferase plasmid. Columns, means of the relative luciferase activity from 4 independent experiments. Luciferase activity in transfected cells treated with vehicle is arbitrarily set as 1.0 ; bars, SE. ${ }^{*} \mathrm{P}<0.05$, for cells treated with vehicle vs. $1 \mathrm{nM}$ of E2 $\beta$.

promoter activity (Fig. 4B), indicating that the biphasic effects of E2 $\beta$ on induction of Cyclin D1 expression occur through regulation of its promoter activity. The Cyclin D1 promoter activity induced by $1 \mathrm{nM} \mathrm{E} 2 \beta$ was inhibited by the Src inhibitors PP2 and dasatinib but weakly by the EGFR inhibitor AG1478 (Fig. 4B).

To further confirm the involvement of Src in estrogeninduced Cyclin D1 expression, these ER-negative breast cancer cells were co-transfected with the Cyclin D1 promoter reporter plasmid and pCMV5/SrcK295M, a dominant- negative mutant of Src or pCMV5/SrcY527F, constitutively active mutant of Src. We found that co-transfection of the dominant-negative mutant of Src abrogated the Cyclin D1 promoter activity induced by $1 \mathrm{nM}$ estrogen while it had no effect in cells treated with $5 \mu \mathrm{M}$ estrogen (Fig. 4C). On the contrary, the constitutively active mutant of Src restored the Cyclin D1 promoter activity suppressed by $5 \mu \mathrm{M}$ E2 $\beta$ (Fig. 4C). These results indicate that Src plays a role in the biphasic response of Cyclin D1 expression to low and high levels of E2 $\beta$. 
A

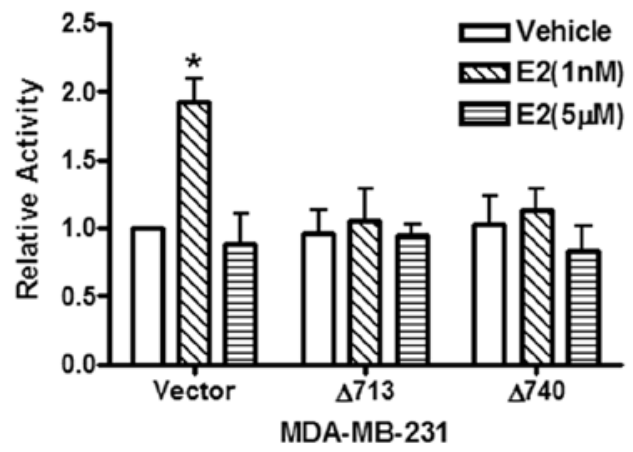

B

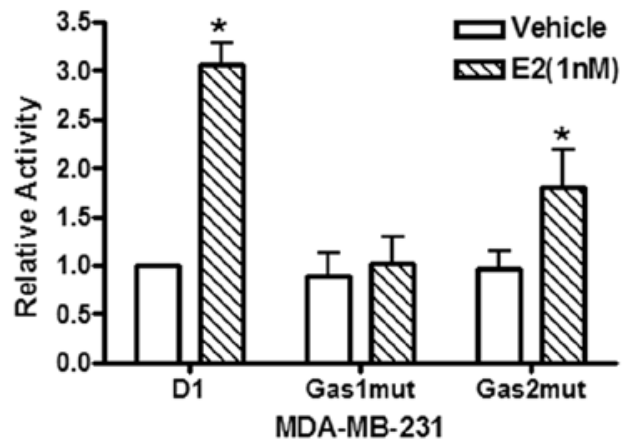

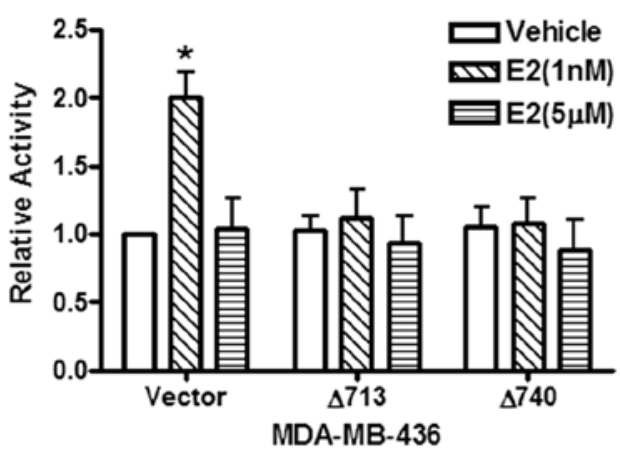

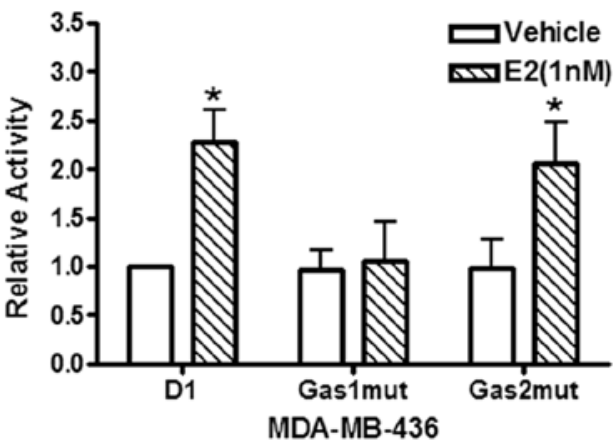

Figure 5. STAT5 is involved in estrogen induction of Cyclin D1 promoter activity. (A) The involvement of STAT5 in E2 $\beta$ induction of Cyclin D1 promoter activity. Cells were transfected with the luciferase reported plasmid Cyclin D1 pl-963 together with an empty expression vector or two dominant-negative STAT5a mutants, STAT5a $\Delta 713$ and STAT5a $\Delta 740$. Transfected cells were treated with vehicle (ethanol), $1 \mathrm{nM}$ or $5 \mu \mathrm{M}$ of E2 $\beta$. Columns, means of the relative luciferase activity from 4 independent experiments. Luciferase activity in transfected cells transfected with a empty expression vector and treated with vehicle is arbitrarily set as 1.0 ; bars, SE. $\mathrm{P}<0.05$, for cells treated with vehicle vs. $1 \mathrm{nM}$ of E2 $\beta$. (B) GAS1 is involved in induction of the Cyclin D1 promoter activity by E2 $\beta$. Cells were transiently transfected with either the wild-type Cyclin D1 promoter (D1) or the same promoter construct containing mutated GAS1 (GAS1mut) or GAS2 (GAS2mut) sequence, respectively. Transfected cells were treated with vehicle or 1 nM of E2 $\beta$ and the luciferase activity was presented relative to the wild-type Cyclin D1 promoter-transfected cells treated with vehicle that is arbitrarily set as 1.0 . * $\mathrm{P}<0.05$, for cells treated with vehicle vs. $1 \mathrm{nM}$ of $\mathrm{E} 2 \beta$.

STAT5 is involved in estrogen-induced Cyclin D1 promoter activity. Previously, it was reported that prolactin activated Cyclin D1 promoter activity through activation of STAT proteins and their interaction with the two consensus $\gamma$-interferon-activation sites (GAS) sites located in the Cyclin D1 promoter (29). We decided to examine whether STAT5 is involved in Cyclin D1 promoter activity induced by $1 \mathrm{nM}$ E2 $\beta$ in these ER-negative breast cancer cells. Two dominant-negative mutants of STAT5a were co-transfected with the Cyclin D1 promoter reporter plasmid and transfected cells were treated with $1 \mathrm{nM} \mathrm{E} 2 \beta$. We found that inclusion of both STAT5a mutants strongly suppressed the Cyclin D1 promoter activity induced by $1 \mathrm{nM}$ E2 $\beta$ (Fig. 5A), suggesting that low concentrations of E2 $\beta$ induced the Cyclin D1 promoter activity through the Src/EGFR/STAT5 pathway in these ER-negative breast cancer cells.

The human Cyclin D1 promoter harbors binding sites for a number of transcription factors. There are two consensus GAS sites located at -457 and -224 of the Cyclin D1 promoter region (relative to the transcription initiation site) that are sites for STAT protein binding (29). To assess the involvement of the two GAS sequences in estrogen-induced Cyclin D1 promoter activity, we transfected these ER-negative breast cancer cells with the Cyclin D1 promoter/luciferase construct containing mutated GAS1 (GAS1mut) and GAS2mut that mutated the two
GAS sequences located at -457 and -224 , respectively. The promoters containing the GAS1 mutation failed to respond to $1 \mathrm{nM}$ E2 $\beta$ while the GAS2 mutation had no effect (Fig. 5B), indicating that the GAS1 site is involved in estrogen-induced Cyclin D1 promoter activity.

ER-a36 is involved in biphasic estrogen signaling of ER-negative breast cancer cells. Previously, we demonstrated that ER- $\alpha 36$ mediates mitogenic estrogen signaling in ER-negative breast cancer MDA-MB-231 and MDA-MB-436 cells using shRNA method; the cells with knocked-down levels of ER- $\alpha 36$ expression failed to exhibit mitogenic responses to estrogen such as activation of MAPK/ERK and induction of Cyclin D1 expression (10). We decided to examine whether ER- $\alpha 36$ is also involved in biphasic estrogen signaling. MDA-MB-231 and MDA-MB-436 cells were transfected with an empty expression vector or an expression vector for shRNA specific for ER- $\alpha 36$ (10). We found that cells with knockeddown ER- $\alpha 36$ expression failed to respond to different concentrations of E2 $\beta$ while control cells transfected with the empty expression vector retained the biphasic estrogen signaling (Fig. 6A), indicating that ER- $\alpha 36$ is involved in the biphasic estrogen signaling in these cells. We further found that 1 nM E2 $\beta$ treatment failed to induce Src-Y416 and EGFR-Y845 phosphorylation in MDA-MB-231 and MDA-MB-436 cell 
A

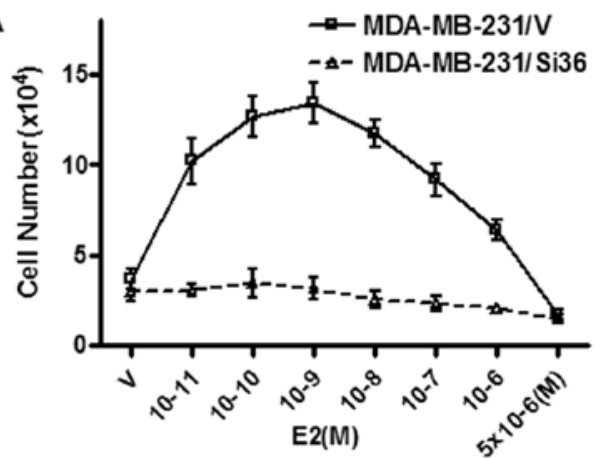

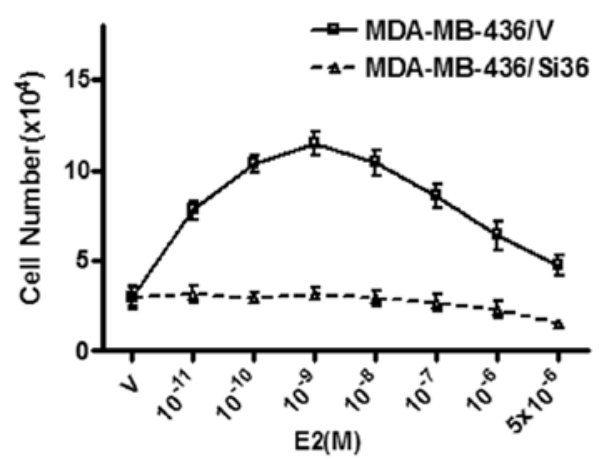

B

C
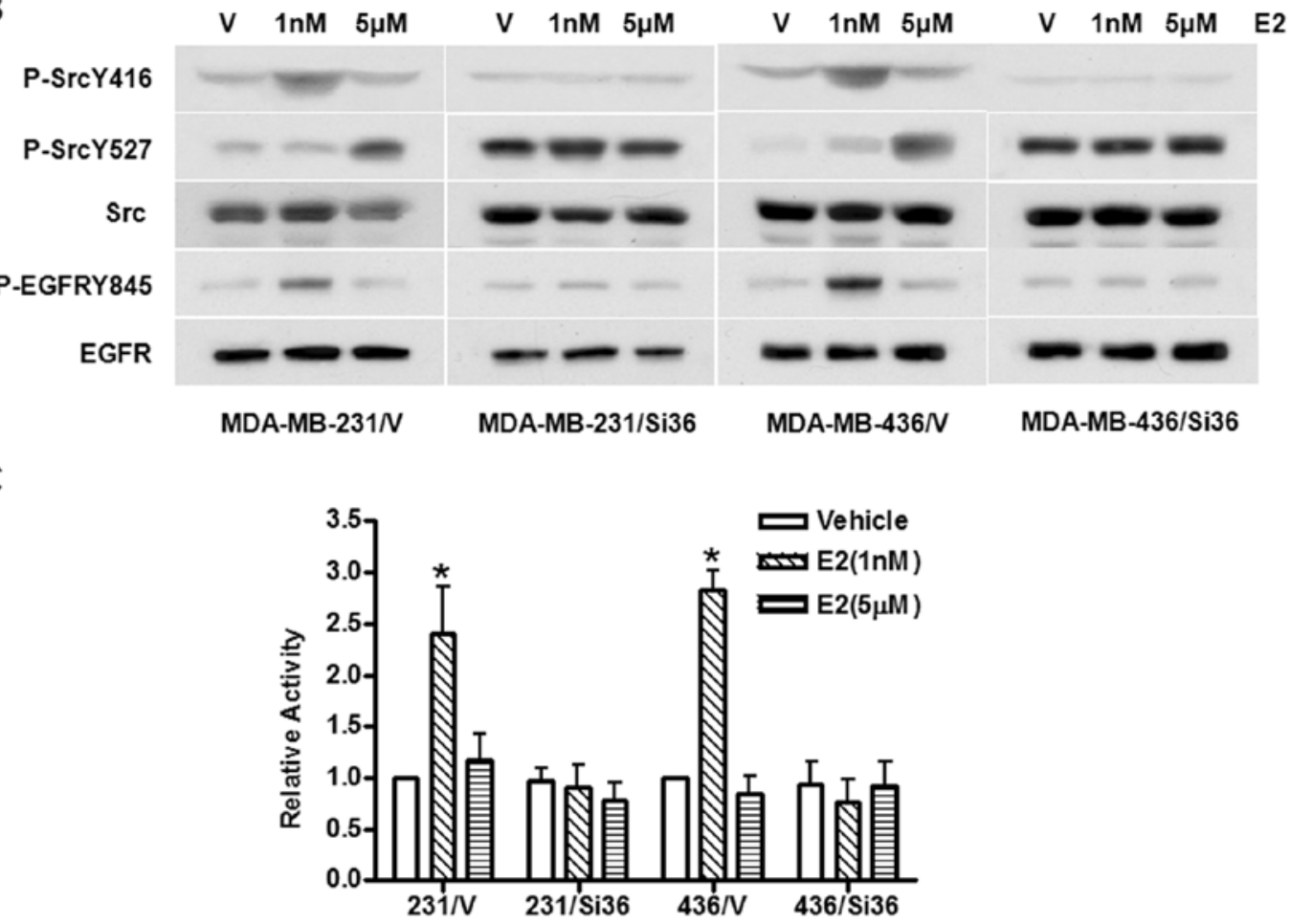

Figure 6. ER- $\alpha 36$ mediates biphasic estrogen signaling in ER-negative breast cancer cells. (A) The effects of E2 $\beta$ on the proliferation rate of MDA-MB-231 and MDA-MB-436 cells with or without ER- $\alpha 36$ expression knock-down. Cells maintained for 3 days in phenol red-free DMEM plus $2.5 \%$ dextran-charcoalstripped fetal calf serum were treated with indicated concentrations of E2 $\beta$ or ethanol vehicle as a control. The cell numbers were determined using an automatic cell counter after 12 days. Five dishes were used for each concentration and experiments were repeated 3 times. The mean cell number \pm SE are shown. (B) Western blot analysis of the effects of $1 \mathrm{nM}$ or $5 \mu \mathrm{M}$ of E2 $\beta$ on the phosphorylation levels of the Src-Y416, Src-Y527 and EGFR-Y845 in MDA-MB-231 and MDA-MB-436 cells. (C). Cells were transfected with the luciferase reporter plasmid driven by the wild-type Cyclin D1 promoter and transfected cells were treated with vehicle (ethanol), $1 \mathrm{nM}$ or $5 \mu \mathrm{M}$ of E2 $\beta$. Columns, means of the relative luciferase activity in transfected cells treated with vehicle that is arbitrarily set as 1.0 from 3 independent experiments; bars, SE. " $\mathrm{P}<0.05$, for cells treated with vehicle (V) vs. $1 \mathrm{nM}$ of E2 $\beta$.

lines carrying ER- $\alpha 36$ expression knocked-down (Fig. 6B). However, the basal levels of Src-Y527 phosphorylation were dramatically increased in MDA-MB-231 and -436 cells transfected with the ER- $\alpha 36$ shRNA expression vector compared to control cells, and treatment of $\mathrm{E} 2 \beta$ at $5 \mu \mathrm{M}$ failed to further increase levels of phosphorylation at Src-Y527 (Fig. 6B). We also tested whether E2 $\beta$ was able to induce Cyclin D1 promoter activity in the cells with ER- $\alpha 36$ knock-down. E2 $\beta$ at $1 \mathrm{nM}$ failed to induce Cyclin D1 promoter activity (Fig. 6C), consistent with our previous report that ER- $\alpha 36$-mediated induction of Cyclin D1 expression by E2 $\beta$ in these cells (10). Together, these results suggested that ER- $\alpha 36$ is involved in biphasic estrogen signaling in ER-negative breast cancer cells.

\section{Discussion}

It is prevailingly thought that mitogenic estrogen signaling does not contribute to development and progression of ER-negative breast cancer that lacks ER- $\alpha 66$ expression. However, early findings demonstrated that ovariectomy can prevent development of both ER-positive and -negative breast cancers $(30,31)$. BRCA1 mutation-related breast tumors, most of which lack ER- $\alpha$ expression, are also effectively prevented by prophylactic ovariectomy $(32,33)$. These findings strongly indicate that mitogenic estrogen signaling is involved in development of some types of ER-negative breast cancer. Previously, Tsai et al reported that E2 $\beta$ was able to induce 
the PI3K/AKT phosphorylation in ER-negative breast cancer MDA-MB-231 cells (34) and malignant growth of ER-negative breast cancer MDA-MB-231 cells was stimulated by estrogen in immunodeficient mice (35). Together, these previous reports indicated that non-genomic and mitogenic estrogen signaling may be retained in some ER-negative breast cancer cells.

Recently, we reported that E2 $\beta$ stimulated proliferation of ER-negative breast cancer cells through ER- $\alpha 36$, a variant of ER- $\alpha$ (10). Here, we used ER-negative breast cancer MDA-MB-231 and MDA-MB-436 cells to study molecular mechanisms underlying biphasic estrogen signaling. These cells were chosen to perform our studies for several reasons. First, these cells lack expression of ER- $\alpha 66$ but highly express ER- $\alpha 36$ (10), which eliminates the possible involvement of ER- $\alpha 66$. Secondly, although MDA-MB-231 cells express low levels of full-length ER- $\beta$, MDA-MB-436 cells express undetectable levels of full-length ER- $\beta$ (10), which would then exclude ER- $\beta$ effects. Thirdly, these cells exhibit a typical biphasic response curve of estrogen signaling (10), which allowed us to study the molecular mechanisms underlying the biphasic estrogen signaling.

The data presented here in confirm that E2 $\beta$ at higher concentrations fails to stimulate proliferation of ER-negative breast cancer cells, which is also consistent with a previous report that pharmacological concentrations of E2 $\beta$ inhibit growth of ER-negative cells (36). We also observed that at high concentrations, E2 $\beta$ failed to activate the MAPK/ERK pathway and to induce the growth-promoting gene Cyclin D1. Thus, activation of the MAPK/ERK signaling and Cyclin D1 expression mediated by non-genomic estrogen signaling also exhibited a biphasic pattern, which provided a molecular explanation to the observed biphasic mitogenic estrogen signaling.

To probe the underlying mechanisms of the biphasic estrogen signaling, we found that at $1 \mathrm{nM}, \mathrm{E} 2 \beta$ induced phosphorylation of Src at Tyr-416, consistent with our previous report that E2 $\beta$ elicits Src-Y416 phosphorylation in these ER-negative breast cancer cells (10). Intriguingly, we found that $5 \mu \mathrm{M}$ of E2 $\beta$ triggered the phosphorylation of Src-Y527. The Src protein has three major domains, SH2 (for Src homology 2), SH3 and the kinase catalytic domain. Both SH2 and $\mathrm{SH} 3$ play a role in protein-protein interactions, while the kinase catalytic domain contains the kinase active site. Src can be switched from an inactive to an active state through control of its phosphorylation state. Src-Y416 can be autophosphorylated, which activates Src by displacing the P-Tyr-416 from the binding pocket, allowing the substrate to gain access. However, phosphorylation of Tyr-527 inactivates Src through the interaction of P-Tyr-527 with the SH2 domain, which effectively folds Src up into a closed, inactive state. Our results thus demonstrated, for the first time, that phosphorylation state of Src-Y416 and -Y527 acts as a switch to turn on and off nongenomic estrogen signaling depending on concentrations of estrogen.

At present, not much is known about how different concentrations of estrogen induce phosphorylation of either Tyr-416 or Tyr-527. Previously, we reported that E2 $\beta$ induced the physical interaction between ER- $\alpha 36$ and Src, and consequently the auto-phosphorylation of Src-Y416 in these ER-negative breast cancer cells (10). Here, we found that ER- $\alpha 36$ knock-down diminished the Src-Y416 phosphorylation induced by $1 \mathrm{nM}$ E2 $\beta$, indicating that ER- $\alpha 36$ is involved in the autophosphorylation of Src-Y416 induced by low concentrations of E2 $\beta$. However, the cells with knocked-down levels of ER- $\alpha 36$ expression exhibited high basal levels of Src-Y527 phosphorylation, which was not further induced by $5 \mu \mathrm{M}$ E2 $\beta$, indicating that abrogation of ER- $\alpha 36$ activity increased basal levels of Src-Y527 phosphorylation and silenced Src activity, consistent with the findings that these ER-negative breast cancer cells with ER- $\alpha 36$ knock-down failed to form xenograft tumors (10). Src-Y527 is a critical site for regulation of Src activity, which can be phosphorylated and dephosphorylated by various proteins $(37,38)$, such as CSK kinase (phosphorylates) or SHP-1 phosphorylase (dephosphorylates). Thus, the phosphorylation state of Src-Y527 is dynamically and strictly regulated by phosphorylation and dephosphorylation. At present, it can only be speculated that different concentrations of estrogen may trigger different conformations of ER- $\alpha 36$, which will then activate either the kinase or the phosphatase to regulate Src-Y527 phosphorylation. On other hand, it is also possible that high concentrations of estrogen may simply inactivate ER- $\alpha 36$, which then increases the basal levels of Src-Y527 phosphorylation to silence Src as we observed in ER- $\alpha 36$ knock-down cells.

We also showed that at low concentrations, E2 $\beta$ induced Src-mediated phosphorylation of the EGFR-Y845 residue, and that $1 \mathrm{nM}$ E2 $\beta$ induced STAT5 mediated activation of Cyclin D1 promoter activity while $5 \mu \mathrm{M}$ of E2 $\beta$ failed to do so, which provided a molecular explanation for the loss of the growth promoting activity observed in cells treated with high concentrations of estrogen. Previously, it was reported that in ER-positive breast cancer T47D cells, estrogen-induced Src activation and Src-dependent phosphorylation of EGFR-Y845 recruits STAT5 as a downstream effector of phosphorylated EGFR-Y845 to induce c-Myc and Cyclin D1 expression (27). Based on these findings and our observations, we speculated that similarly to ER-positive breast cancer cells, ER- $\alpha 36$ mediates mitogenic signaling of low-concentration estrogen through the EGFR/Src/STAT5 pathway in ER-negative breast cancer cells.

In summary, we have shown that ER-negative breast cancer cells exhibited biphasic estrogen signaling and that Src acts as a switch to turn on/off non-genomic estrogen signaling mediated by ER- $\alpha 36$. As a novel player in estrogen signaling, ER- $\alpha 36$ is involved in mitogenic estrogen signaling induced by physiological concentrations of estrogen through the Src/EGFR/STAT5 pathway. High-doses or pharmacological concentration of estrogen were used to treat breast cancer before the introduction of the antiestrogen, tamoxifen. Recently, laboratory models of resistance to antiestrogens such as tamoxifen demonstrated that estrogen functions to inhibit cell growth and induce apoptosis in these ER-positive breast cancer cells with acquired antiestrogen resistance (21-26). Our results here thus provide a strong rational for clinical evaluation of high-dose estrogen in ER-negative breast cancer.

\section{Acknowledgements}

This study was supported by NIH grantDK070016 and Nebraska Tobacco Settlement Biomedical Research Program Awards 
(LB-595) to Z.-Y.W. We thank Dr Yun Qiu, Dr Linda Schuler, Dr Hiroko Yamashita and Dr Chris Albanese for providing the constructs containing Src mutants, Cyclin D1 promoter GAS1 and 2 mutants, STAT5 mutants and the wild-type Cyclin D1 promoter.

\section{References}

1. Nilsson S, Makela S, Treuter E, et al: Mechanisms of estrogen action. Physiol Rev 81: 1535-1565, 2001.

2. Kelly MJ and Levin ER: Rapid actions of plasma membrane estrogen receptors. Trends Endocrinol Metab 12: 152-156, 2001 .

3. Segars JH and Driggers PH: Estrogen action and cytoplasmic signaling cascades. Part I: membrane-associated signaling complexes. Trends Endocrinol Metab 13: 349-354, 2002.

4. Wang Z, Zhang X, Shen P, Loggie BW, Chang Y and Deuel TF: Identification, cloning, and expression of human estrogen receptor- $\alpha 36$, a novel variant of human estrogen receptor- $\alpha 66$. Biochem Biophys Res Commun 336: 1023-1027, 2005.

5. Wang Z, Zhang X, Shen P, Loggie BW, Chang Y and Deuel TF: A variant of estrogen receptor- $\alpha$, hER- $\alpha 36$ : transduction of estrogen- and antiestrogen-dependent membrane-initiated mitogenic signaling. Proc Natl Acad Sci USA 103: 9063-9068, 2006.

6. Zou Y, Ding L, Coleman M and Wang Z: Estrogen receptor-alpha (ER- $\alpha$ ) suppresses expression of its variant ER- $\alpha 36$. FEBS Lett 583: 1368-1374, 2009.

7. Lee LM, Cao J, Deng H, Chen P, Gatalica Z and Wang ZY: ER- $\alpha 36$, a novel variant of ER- $\alpha$, is expressed in ER-positive and -negative human breast carcinomas. Anticancer Res 28: 479-483, 2008.

8. Shi L, Dong B, Li Z, et al: Expression of ER- $\alpha 36$, a novel variant of estrogen receptor $\alpha$, and resistance to tamoxifen treatment in breast cancer. J Clin Oncol 27: 3423-3429, 2009.

9. Vranic S, Gatalica Z, Deng H, et al: ER- $\alpha 36$, a novel isoform of ER- $\alpha 66$, is commonly over-expressed in apocrine and adenoid cystic carcinomas of the breast. J Clin Pathol 64: 54-57, 2011.

10. Zhang XT, Kang LG, Ding L, Vranic S, Gatalica Z and Wang ZY: A positive feedback loop of ER- $\alpha 36 /$ EGFR promotes malignant growth of ER-negative breast cancer cells. Oncogene 30: 770-780, 2011

11. vom Saal FS, Timms BG, Montano MM, et al: Prostate enlargement in mice due to fetal exposure to low doses of estradiol or diethylstilbestrol and opposite effects at high doses. Proc Nat Acad Sci USA 94: 2056-2061, 1997.

12. Miodini P, Fioravanti L, Di Fronzo G and Cappelletti V: The two phyto-oestrogens genistein and quercetin exert different effects on oestrogen receptor function. Br J Cancer 80: 1150-1155, 1999.

13. Li L, Andersen ME, Heber S and Zhang Q: Non-monotonic dose-response relationship in steroid hormone receptor-mediated gene expression. J Mol Endocrinol 38: 569-585, 2007.

14. Calabrese EJ: Estrogen and related compounds: biphasic dose responses. Crit Rev Toxicol 31: 503-515, 2001.

15. Kuchler RJ and Grauer RC: Effects of natural estrogens on L strain fibroblasts in tissue culture. Proc Soc Exp Biol Med 110: 287-292, 1962.

16. Riley PA, Latter A and Sutton PM: Reversible morphological changes in human breast fibroblasts exposed to stilboestrol. Eur J Cancer 14: 579-586, 1978.

17. Hass R and Sohn C: Effects of estrogen on cell cycle progression and associated metabolic pathways in normal and tumorigenic breast cells. Signal Transduction 3: 9-16, 2003.

18. Rao PN and Engelberg J: Structural specificity of estrogens in the induction of mitotic chromatid non-disjunction in HeLa cells Exp Cell Res 48: 71-81, 1967.
19. Tsutsui T, Maizumi H, McLachlan JA and Barrett JC: Aneuploidy induction and cell transformation by diethylstilbestrol: a possible chromosomal mechanism in carcinogenesis. Cancer Res 43: 3814-3821, 1983.

20. Watson CS, Jeng YJ and Kochukov MY: Nongenomic signaling pathways of estrogen toxicity. Toxicol Sci 115: 1-11, 2010.

21. Wolf DM and Jordan VC: A laboratory model to explain the survival advantage observed in patients taking adjuvant tamoxifen therapy. Recent Results Cancer Res 127: 23-33, 1993.

22. Yao K, Lee ES, Bentrem DJ, et al: Antitumor action of physiological estradiol on tamoxifen-stimulated breast tumors grown in athymic mice. Clin Cancer Res 6: 2028-2036, 2000.

23. Osipo C, Gajdos C, Liu H, Chen B and Jordan VC: Paradoxical action of fulvestrant in estradiol-induced regression of tamoxifen-stimulated breast cancer. J Natl Cancer Inst 95: 1597-1608, 2003.

24. Song RX, Mor G, Naftolin F, et al: Effect of long-term estrogen deprivation on apoptotic responses of breast cancer cells to 17 $\beta$-estradiol. J Natl Cancer Inst 93: 1714-1723, 2001.

25. Lewis JS, Osipo C, Meeke K and Jordan VC: Estrogen-induced apoptosis in a breast cancer model resistant to long-term estrogen withdrawal. J Steroid Biochem Mol Biol 94: 131-141, 2005.

26. Lewis JS, Meeke K, Osipo C, et al: Intrinsic mechanism of estradiol-induced apoptosis in breast cancer cells resistant to estrogen deprivation. J Natl Cancer Inst 97: 1746-1759, 2005.

27. Fox EM, Bernaciak TM, Wen J, Weaver AM, Shupnik MA and Silva CM: Signal transducer and activator of transcription $5 \mathrm{~b}, \mathrm{c}-\mathrm{Src}$, and epidermal growth factor receptor signaling play integral roles in estrogen-stimulated proliferation of estrogen receptor-positive breast cancer cells. Mol Endocrinol 22: 1781-1796, 2008

28. Yamashita H, Iwase H, Toyama T and Fujii Y: Naturally occurring dominant-negative Stat5 suppresses transcriptional activity of estrogen receptors and induces apoptosis in T47D breast cancer cells. Oncogene 22: 1638-1652, 2003.

29. Brockman JL, Schroeder MD and Schuler LA: PRL activates the cyclin D1 promoter via the Jak2/Stat pathway. Mol Endocrinol 16: 774-784, 2002.

30. Early Breast Cancer Trialists' Collaborative Group: Systemic treatment of early breast cancer by hormonal, cytotoxic, or immune therapy. 133 randomised trials involving 31,000 recurrences and 24,000 deaths among 75,000 women. Early Breast Cancer Trialists' Collaborative Group. Lancet 339: 1-15, 1992.

31. Nissen-Meyer R: 'Prophylactic' ovariectomy and ovarian irradiation in breast cancer. Acta Unio Int Contra Cancrum 20: $527-530,1964$

32. Narod SA: Hormonal prevention of hereditary breast cancer. Ann NY Acad Sci 952: 36-43, 2001

33. Rebbeck TR, Levin AM, Eisen A, et al: Breast cancer risk after bilateral prophylactic oophorectomy in BRCA1 mutation carriers. J Natl Cancer Inst 91: 1475-1479, 1999.

34. Tsai EM, Wang SC, Lee JN and Hung MC: Akt activation by estrogen in estrogen receptor-negative breast cancer cells. Cancer Res 61: 8390-8392, 2001.

35. Friedl A and Jordan VC: Oestradiol stimulates growth of oestrogen receptor-negative MDA-MB-231 breast cancer cells in immunodeficient mice by reducing cell loss. Eur J Cancer 30A: 1559-1564, 1994.

36. Nomoto S, Arao Y, Horiguchi H, Ikeda K and Kayama F: Oestrogen causes $\mathrm{G} 2 / \mathrm{M}$ arrest and apoptosis in breast cancer cells MDA-MB-231. Oncol Rep 9: 773-776, 2002.

37. Chong YP, Mulhern TD and Cheng HC: C-terminal Src kinase (CSK) and CSK-homologous kinase (CHK) - endogenous negative regulators of Src-family protein kinases. Growth Factors 23: 233-244, 2005.

38. Playford MP and Schaller MD: The interplay between Src and integrins in normal and tumor biology. Oncogene 23: 7928-7946, 2004. 